\title{
Research on Modeling and Simulation of Agent-based Intelligent Teaching System
}

\author{
Yang Yang \\ Information Center, Hubei Polytechnic Institute, Xiaogan, China
}

Email address:

muyiee@163.com

\section{To cite this article:}

Yang Yang. Research on Modeling and Simulation of Agent-based Intelligent Teaching System. Science Journal of Education . Vol. 8, No. 1, 2020, pp. 27-31. doi: 10.11648/j.sjedu.20200801.15

Received: February 29, 2020; Accepted: April 2, 2020; Published: April 14, 2020

\begin{abstract}
With the development of information technology such as Artificial Intelligent and mobile computing, various kinds of education concepts or products have become a reality which great promote the development of educational innovation. Nowadays more and more attention are put on the reform of teaching mode, such as MOOC, SPOC, Micro-Course and so on. These teaching modes have tremendous advantages over traditional teaching on facilitating the students' study, enhancing their learning interest and broadening their horizons. However, the problem of lacking of platform support for the integration of these teaching models still exists. In order to solve this problem, this article propose a model of intelligent teaching system, named ITS for short. The ITS could be regarded as a platform or a system but also a new generation of innovation teaching environment, consisting of software platform and hardware devices. Compared with the traditional classroom teaching, the intelligent teaching system gives emphasis on virtual teaching online and offline, in and out of class. In terms of the definition listed above, this article presents a modeling and simulation of intelligent teaching system basing on agent technique, targeting all components concerned, such as knowledge base, network education system, intelligent teaching platform and some hardware devices. The simulation proves that the modeling of the intelligent teaching system is an efficient way to enhance the quality of teaching and improve the teaching effect, and the intelligent teaching system has the potential to become an application product in the market.
\end{abstract}

Keywords: Intelligent Teaching, Virtual Teaching, Agent, Modeling and Simulation

\section{Introduction}

With the development of Artificial Intelligent and mobile computing, the deep integration of education teaching and information technology becomes a new trend for education reform in new era. Various information techniques are widely used in the area of teaching, such as Artificial Intelligent technique which is applied for analyzing and evaluating the teaching effect in terms of the data collected by the video monitors installed in the classroom. What's more, teachers and students use the mobile phones to interact and communicate with each others, sharing their attitude about the degree of course difficulty, uploading the photo copies of class exercise to the cloud servers, and submitting the class tests and exercises through mobile application programs.

New technology generates new teaching modes. Basing on these new techniques, flipped classroom [1], a new pedagogical method emphasizing on combining the video teaching and classroom teaching together, and giving the self-study more priority than teaching, is at the center of the attention and discussion about how to share and broadcast the knowledge in the world quickly and freely. However, in the new era of high technology, what the educators pursue is not merely using flipped classroom to catalyzing change of the traditional scene, but a platform or a system to exercise all types of teaching mode, thus enhancing the teaching effect and helping the students establish the knowledge framework and architecture, and finally reaching the teaching goals. Conclusively, we define such a platform or system as intelligent teaching system (ITS), which essentially is an environment, contains hardware devices to collect teaching data, and software platform to support the online-study as well as the active, group-based teaching activities.

ITS is not a new conception but derived from the wisdom classroom or intelligent classroom. However, the intelligent classroom is more likely to Internet of Things (IoT), 
especially integrating environmental conditioning, video monitoring and remote control together to promoting the modernized teaching. Different from the intelligent classroom, ITS is more likely to using software system related to facilitate students' study, such as the supporting software platform of MOOC, SPOC, Micro-Course Online Video, etc.

Although ITS has an approximate definition and a general range, more detailed and accurate work is needed to be done in our research job. As we would like to transfer the ITS conception in the laboratory to application products in the market, a detailed complete report of ITS undoubtedly has the highest priority. In the article, we use the agent-based technique to analyse the ITS, and much work is put on the modeling and simulation of the ITS.

\section{The Definition of Intelligent Teaching System}

The intelligent teaching system (ITS) is a new generation of teaching assistance platform, targeting at more intelligent and more functional roles, focusing at establishing the teaching platform, constructing the teaching scene in live style, analyzing the teachers' and students' behaviour [2], recommending the study material [3] and so on. The more detailed definition is given as below.

From the perspective view of software system, ITS has various functionality. First of all, it's a gathering place of teaching videos, as well as teaching resources. Through ITS, resources like audios, videos, documents, and electronic papers are managed in digital database. People operate the database freely through website interface, and the operation orders like ADD and DELETE are transferred on the Internet. Secondly, ITS has the function as the MOOC class has, which means students could watch the video online and offline, any time any where. Thirdly, in terms of the study behaviour of the students, recorded in the MOOC website, ITS could analyze the study status about the students, and give suggestions and recommendations from a data point of view.

From the hardware perspective, ITS contains several devices, such as video cameras, sound collectors, mobile phones and pads, etc. Firstly, the cameras are used to collect the data about facial expression, body movements, and sitting postures to gauge whether the attention and the learning attitude is good enough. This process definitely requires high technology, including video processing, image analyzing, and image understanding, and finally AI. When all these techniques are integrated together into hardware chips, captured footage through video cameras are transferred from visual scene to understandable data easily and quickly. Secondly, apart from camera equipment, the sound collectors installed in the classroom could identify the enthusiasm and the engagement through the size of the sound by students. When the atmosphere in classroom is intense and positive, teachers and students are more likely in a interactive teaching session, and the frequency and amplitude of the sound is quite different from the ordinary scene. However, the process of analysis and judgement by voice also needs mounts of data training which requires AI technique. Thirdly, in the mobile age, mobile phones and pads are involved during lessons too, via which students and teachers communicating with each other, discussing and interacting. Sometimes these mobile devices are treated as file transfer managers to upload the photo copies of exercise and test papers to teachers' sides, and sometimes they are terminal devices of students which have abundant and flexible functionality for discussions and reporting.

\section{The Agent Technique}

The agent technique originally appeared in the field of artificial intelligence in the 1970s, as to solve the complex problems which are hard to predict. There are many call names about the agent, such as proxy, subject, intelligent agent and so on. The agent technique represents a series methods to communicate with the environment, for the agent itself has the properties of perception, problem-solving ability, bidirectional communication capability, etc. Theoretically, we call the agent an intelligent entity which has an aptitude for continuously getting intellisense, acting autonomously, changing dynamically, and owing the ability of building up its own knowledge framework, to predict the environment status and deduce the solution. On the basis of description of Wooldridge's definition [4], the agent has the following properties:

1) autonomy: agents act without total instructions from external environment, and have certain controls over their own actions and internal states.

2) social ability: agents could communicate with each other by some kind of special language, as to establish the channels among agents.

$3)$ reactivity: agents are able to perceive the external and internal changes in the life cycle, and response in real time to adapt to the environment.

4) pro-activeness: agents take actions in an initiative and goal-directed mode, rather than simple and mechanical response to the environment.

Except for the "weak" definition made for the agent, it has a "strong" one, which describes the agents originally applied to human beings, such as knowledge, belief, intention, and obligation. What's more, some other attributes, for example, mobility and rationality are always mentioned in the context of intelligent environment. The more detailed descriptions about these features are listed below [5]:

1) knowledge: information and understanding that gained from the environment or self experience.

2) belief: opinions or believes which urge the agents to compute and make a choice about the next step in various condition. Definitely it could be updated by the sensors in terms of the environment change.

3) intention: a will or goal affected by the belief of the agent, it directs the agent to act in a certain way in a 
specific direction to resolve problems.

4) obligation: a course of actions that agent is required to take to finish its duty.

5) mobility: an attribute of agent to simulate its actions moving from one place to another in the system modeling.

6) rationality: a predefined assumption that agent will think like humans being reasonable, especially being accordant with its actions.

As stated above all, the agent is a computable entity in the area of system modeling and simulation. It has several human-like features so as to communicate with each other, living in the society composed by groups of agents, interacting with the environment by input and output, and finishing tasks or goals in time and in orders. Generally, we call this process ABM (agent based modeling) [6], ABS (agent based simulation), MAS (multi-agent simulation) or MABS (multi-agent based simulation), although there are differences among these concepts.

\section{The Agent Based Modeling Of ITS}

There are several ways of modeling in the field of software engineering, and the agent based modeling is developed recently, having a lot of researchers devoting into this direction. However, although the agent approach is hot and much work has been done, it still deserves to be further discussed. As we all know, the agent technique is a new intelligent distributed computing technology, accompanied with two characters. For one thing, the ABM technique applies AI theory for problem modeling, assuming that the study objects having the human-like properties [7], putting emphasis on autonomy and intelligence. For another thing, the concepts originally used in the area of distributed system [8], such as mobility, security and cooperative, also become important research directions in ABM. In this article, basing on these two characters, a detailed description of the modeling process of ITS is listed.

\subsection{Architectural Model of ITS}

As discussed in the section II, the ITS involves two aspects, hardware and software. In other words, it establishes on the basis of the hardware equipment, and takes full advantage of software functionality. The hardware part mainly puts emphasis on sensing the environment, detecting the exception, and collecting the key data. Correspondingly, the software part provides the human - computer interface, analyzing actions and behaviors of the users, and making scientific predication. To have a full cognition of ITS, the hardware component is divided into video agents, sound agents and PDA agents which including mobile phones as well as pads, etc. And the software component is something like MOOC system, consisting of student agent, teacher agent, administrator agent and so on. The following picture gives the architecture of the whole ITS.

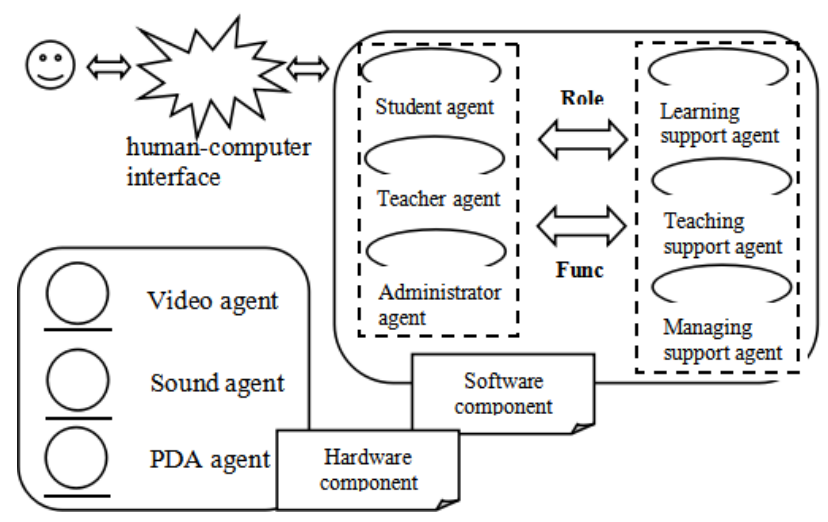

Figure 1. Architecture of the whole ITS.

\subsection{Modeling of the Software Component of ITS}

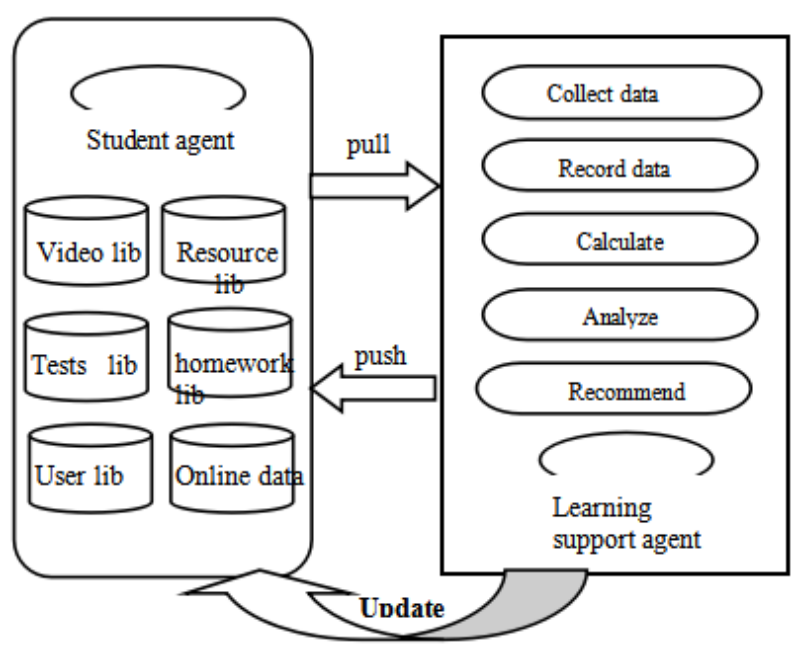

Figure 2. The student agent and learning support agent.

As shown in the figure 1, the software component of ITS is roughly divided into several groups. The first group consists of student agent [9] and learning support agent [10]. These two kinds of agents have similar belief and intention which emphasizing on facilitating study smoothly and have complementary actions. The student agent may usually refer to a course learner, or a beginner in a new academic area. It has great interest on mastering the knowledge, longing for being a domain expert. As an actor, the student agent logs into the website system, managing its basic private information, setting security and system options, and watching the video resources provided by the teacher agent. This scene description about online study process greatly reforms the traditional classroom teaching. As a result, a new relationship conception in education focusing on online to offline is established. Since the early model of MOOC course has the similar concepts to some extent, the learning support agent has to be mentioned to differentiate from it. Generally, the learning support agent has a tool box, which has lots of functionality, especially on learning data collecting, sorting, calculating, analyzing and recommending. For example, the most obligations of the learning support agent are mentioned as follows: recording the online time and learning progress of the students, maintaining the lists of sign ups every day, 
counting the homework scores or tests scores in every sections, pushing the related materials to students. In order to give a more detailed and visual outline, figure 2 is demonstrated. As shown, several databases are related to the student agent, proximate the most information about the learners. Take the online data library for example, all the data collected in the watching process are sent into the process chain of the learning support agent. After that, if data are updated, the online data library will be refreshed. This loop could be called an iteration, which means the high quality of studying effect is being accumulated.

The second group consists of teacher agent and teaching support agent [11]. The combination of these two types of agents could be called teaching model or teacher assistant either. As the purpose it is originally designed for, these two agents are well prepared to provide help with lesson planning, resources arranging, teaching organization and interaction, etc. When the teacher logs on from the system interface, he or she then owns the role and permissions of the teacher agent. However, the teacher agent needs to communicate with the teaching support agent, to finish a series of tasks or jobs. In order to understand this process fully, we would firstly divide the teaching support agent into a few of sub agents, such as Lesson Planning Agent (LPA), helping teachers prepare lessons; Teaching Process Management Agent (TPMA) [12], responsible for teacher-student interaction, using some equipments; Group Agent (GA), used for group discussion and learning; Homework Agent (HA), facilitating correcting students' homework; Test Agent (TA), for having class tests. These agents are not independent, they are working in coordination with each other in terms of some algorithms [13]. The most common algorithm is called BLACKBOARD [14], which would establish a distributed collaborative decision support mechanism to address complex problems. Since the teaching activities are processes requiring sufficient materials such as videos, audios or electronic documents, the libraries or databases of these resources are also built up. When all the components are prepared well, let's take the process of lessons preparation before class for example to further understand the execution mechanism of the second group. Firstly, the teacher would search whether other teachers have done the same courses on the website with the help of LPA, so as to duplicate or move the materials created by others into his/her own class libraries, avoid repetitive tasks. Secondly, the teacher would upload the prepared courseware into the specified syllabus, as well as some video or audio clips. These materials will be pushed to students as the preview before class. Thirdly, by the aid of HA and TA, the class-related homework and tests are stored into the databases respectively. Once needed, the teacher would retrieve these prepared data conveniently and quickly. The other thing to note is that the relationship between the first group and the second group is tight. It's obvious that the student agent would pursue the knowledge information prepared by the teacher agent. They are like Producers-andConsumers in some degree. And the feedback of the student agent would facilitate the teaching quality of the teacher agent.

The third group consists of administrator agent and the managing support agent. These pairs of agents are mainly concerned with the system security management including the account administration, authorization and authentication, and etc. They are primarily serving the system administrators. In this group, security is the most important consideration comparing with other characters.

\subsection{Modeling of the Hardware Component of ITS}

There are three hardware components in the composition of ITS. We call them video agents, sound agents and PDA agents respectively. The video agents represent video cameras or camera equipment. They are often installed in the front of the classroom to capture the teaching scenes. It's quite useful to classroom discipline management. When some students break the classroom rule, the teacher could easily identify them. What's more, the mental intention, facial expression, eye and body movement, and siting position are all becoming data collected by the camera continuously, transferred to the back-end computers. In the same way, the sound collectors represent by the sound agents analyze the frequency and amplitude of the sound in the classroom. Disorderly and unsystematic sound often means bad classroom order and discipline. As a result, a message would sent to the teaching discipline administrators. However, the video cameras and sound collectors only play role in the offline teaching, or the online and offline combination teaching.

The PDA agents often refers to the mobile phones or pads. They are useful and become an important strategy [15] for modern education. The teachers and the students use the mobile phones to establish a virtual discussion groups, sending voice messages and video messages, and delivering study files to each other. Sometimes the mobile phones incorporate with other medias such as TV, especially when broadcasting PPT synchronously among teachers and students. This functionality is quite important when carry out intelligent teaching. However, WIFI signal should be good enough to be the network infrastructure.

\section{Conclusion}

This research study gives a detailed description of the ITS (intelligent teaching system) using the agent based technique. Firstly, a more accurate definition of ITS is presented: for establishing a multifunctional platform or a system to realizing all types of teaching mode, such as MOOC, flipped classroom, and so on. Then using the agent based technique to model the hardware component and software component of ITS. The modeling and simulation process is from whole to parts, focusing the function definition and the scene description. Through evaluation, the modeling of ITS has the potential to translate from conceptual design in the laboratory to application products in the market. 


\section{Acknowledgements}

Acknowledgements are given to the province teaching research project of colleges and universities in hubei province in 2018:"Practice and Research of Integrated Network Teaching Service Platform based on Cloud Architecture" (No. 2018546);

\section{References}

[1] Barbara Kerr, "The flipped classroom in engineering education: A survey of the research," Proceedings of 2015 International Conference on Interactive Collaborative Learning (ICL). 20-24 September 2015, pp. 815-818.

[2] Han, J., Zhao, W., Jiang, Q., Oubibi, M., \& Hu, X. (2019). Intelligent Tutoring System Trends 2006-2018: A Literature Review. 2019 Eighth International Conference on Educational Innovation through Technology (EITT).

[3] Lin H, Xie S, Xiao Z, et al. Adaptive Recommender System for an Intelligent Classroom Teaching Model [J]. International Journal of Emerging Technologies in Learning (iJET), 2019, 14 (05).

[4] Michael Wooldridge, Nicholas R. Jennings, Intelligent agents: theory and practice. The Knowledge Engineering Review, Vol. 10: $2,1995,115-152$.

[5] Yoav Shoham, Agent-oriented programming, Artificial Intelligence 60 (1993) 51-92.

[6] C. Macal and M. North, "Introductory tutorial: Agent-based modeling and simulation," Proceedings of the Winter Simulation Conference 2014, Savanah, GA, 2014, pp. 6-20.
[7] Klügl, F., \&Bazzan, A. L. C. Agent-Based Modeling and Simulation. AI Magazine, 2012, 33 (3), 29.

[8] Cao J, Wang X, Das S K. A framework of using cooperating mobile agents to achieve load sharing in distributed web server groups [J]. Future generation computer systems, 2004, 20 (4): p. 591-603.

[9] Yuan, L., Dai, F., \& Huang, C. (2009). Intelligent Agent-Based Remote Education System. 2009 First International Workshop on Education Technology and Computer Science.

[10] Hong H, Kinshuk D, He X, et al. Application of Mobile agents in Web-based Learning environment [J]. Computer Assisted Instruction, 2001: 7.

[11] Jafari A. Conceptualizing Intelligent Agents For Teaching and Learning [J]. Educause Quarterly, 2002, 25 (3): 28-34.

[12] Baylor, A. L. (2002). Agent-based learning environments for investigating teaching and learning. Journal of Educational Computing Research, 26 (3), 249-270.

[13] Valk J, Witteveen C. Multi-agent Coordination in Planning. PRICAI 2002: Trends in Artificial Intelligence, 7th Pacific Rim International Conference on Artificial Intelligence, Tokyo, Japan, August 18-22, 2002, Proceedings. DBLP, 2002.

[14] Corkill D D. Collaborating Software: Blackboard and Multi-Agent Systems \& the Future [J]. 2003.

[15] G. S. Ntloedibe-Kuswani. Mobile Phones As Support For Distance Learning. IADIS International Conference e-Learning 2008, Amsterdam, The Netherlands, July 22-25, 2008. Proceedings. 